\title{
Open Source Resume
}

\author{
Priya Behlkar ${ }^{1}$, Roshni Gondawale ${ }^{2}$, Bhagyshree Lokare ${ }^{3}$, Snehal Kamble ${ }^{4}$, Prof. A.V. Kanade \\ Student, IT Dept, BVCOE, Pune, India ${ }^{1,2,3,4}$ \\ Professor, IT Dept, BVCOE, Pune, India ${ }^{5}$
}

\begin{abstract}
Open source resume is a web-based application to help software development teams to recruit developers based on their open source system contribution activities. This tool provides insights into developers' Practical contributions to open source system projects, which can be useful criteria for judging whether they are suitable for joining the development team based on their different qualifications. To overcome the Empowerment issues many job portals came into existence even though these portals are trying their best we are facing many difficulties to get response from the corporate side, at the same time even corporate human resource people are facing difficulties to find the employee who fits their requirement. Action is needed to overcome these many challenges and make everything easier for the users.
\end{abstract}

Keywords: Open Source Software; Mining Software Repositories; Developer Expertise; Visualization Tool.

\section{INTRODUCTION}

Recently, job recommendation has attracted a lot of Functions of paper-

research attention and has played an important role on the This paper presents an effective approach for resume online recruiting website. Different from traditional information extraction to support automatic resume recommendation systems which recommend items to management. In the first pass, a resume is segmented into users, job recommender systems recommend one type of a consecutive block attached with labels indicating the users (e.g., job applicants) to another type of users (e.g., information types. Then in the second pass, the detailed recruiters). In particular, job recommender system is information, such as Name and Address, are identified in designed to retrieve a list of job positions to a job certain blocks (e.g. blocks labelled with Personal applicant based on his/her preferences or to generate a list Information), instead of searching globally in the entire of job candidates to a recruiter based on the job resume. The most appropriate model is selected through requirements. To obtain a good recommendation results, experiments for each IE task in different passes. The many recommendation approaches are presented and experimental results show that this cascaded hybrid model applied in the JRS. Typically, given a user, existing JRSs achieves better F-score than flat models that do not apply employ a specific recommendation approach to generatea the hierarchical structure of resumes. It also shows that ranked list of jobs/candidates. However, different users applying different IE models in different passes according may have different characteristics and a single to the contextual structure is effective recommendation approach may not be suitable for all users.

Advantage-

Visualization tool was implemented with another main

Therefore, a high-quality JRS should have the capability of choosing the appropriate recommendation approaches according to the user's characteristic. We proposed an approach to construct and visualize developers' OSS biographies based on their contribution activities.

- Based on the approach, a visualization tool was implemented with another main feature of querying for OSS developers based on user's criteria.

- The tool is considered to provide a convenient way to seek suitable developers for constructing development teams in software development.

\section{LITERATURE SURVEY}

feature of querying for OSS developers based on user's criteria.

Disadvantages-

Advance features are not introduced.

2. S. T. Al-Otaibi and M. Ykhlef, "A survey of job recommender systems, "International Journal of the Physical Sciences, vol. 7(29), pp. 5127-5142, July,2012.

Functions of paper-

Boolean arithmetic is used in this system. Attributes of user are classified into 0 or 1 . Key skills that are required by $\mathrm{HR}$ are represented by 1 else 0 . With the help of this Boolean attribute ranking is made. Higher the rank, better K. Yu, G. Guan and M. Zhou, "Resume information is the customer. extraction with cascaded hybrid model," In Proceedings of the 43rd Annual Meeting of the ACL, pp. 499-506, Ann AdvantageArbor, Michigan, June, 2005.

Manual work of HR is reduced. 
Disadvantages-

Complex arithmetic hence difficult to implement.

3. Yao Lu , Sandy El Helou , Denis Gillet "A Recommender System for Job Seeking and Recruiting website"

Functions of paper-

In this paper, a hybrid recommender system for job seeking and recruiting websites is presented. The various interaction features designed on the website help the users organize the resources they need as well as express their interest. The hybrid recommender system exploits the job and user profiles and the actions undertaken by users in order to generate personalized recommendations of candidates and jobs

\section{Advantage-}

Simple to handle. Saves candidates time.

Disadvantages-

Hectic for HR.

4. Wenxing Hong, Siting Zheng, Huan Wang "A Job Recommender System Based on User Clustering"

Functions of paper-

New concept of clustering was introduced. Cluster can be defines as collection of related data or information. Generalized clusters are formed based on various attributes These clusters are predefined. No new clusters can be added.

\section{Advantage-}

No complex arithmetic is required. Simple to implement.

Disadvantage-

No accuracy is achieved.

5 Xin Yang , ErinaMakhira , Kenji Fujiwara , HajmuLida“'Open Source Resume (OSR): A Visualization Tool for Presenting OSS Biographies of Developers"

Function Of Paper-

Uses the concept of Clustering. Clusters are in more detailed format. New clusters can also be added. Tree structure of clusters is formed.

\section{Advantage-}

Efficient from both sides i.e. companies and employees. No pre-defined clusters.

Disadvantage-

Actual implementation is hectic.

\section{RELATED WORK}

Social Network Service has been one of the mostpopular topics around the world since the last decade [1]. As aresult of the rapid increase in SNS users, some studies applied data mining techniques to analyse Social
Networking Service for exploring trending topics and seeking desirable community for oneself

\section{- Content-based Recommendation}

The principle of a content-based recommendation is to suggest items that have similar content information to the corresponding users. For example, in the recommendation that recommending jobs to a job applicant, the content is the personal information and their job desires. While recommending candidates to recruiters, the job description posted by recruiters, including the background description of enterprises, are used as the content for recommendation.

\section{- $\quad$ Collaborative Filtering Recommendation}

Collaborative filtering recommendation, known as the user-to-user correlation method, finds similar users who have the same taste with the target user and recommends items based on what the similar users like. The key step in CFR is computing the similarities among users. Collaborative filtering recommendation algorithm can be classified into memory-based and model-based. In the memory-based collaborative filtering recommendation, a user-item rating matrix is usually used as the input $[2,3]$. Applied in the job recruiting domain, some user behaviors or actions can generate the user-item rating matrix according to the predefined definitions and transition rules.

\section{- Knowledge-based Recommendation}

In the knowledge-based recommendation, rules and patterns obtained from the functional knowledge of how a specific item meets the requirement of a particular user, are used for recommending items [5]. For example, employees who have one or more years of work experience exhibit better performance as compared to those without experience.

\section{- Reciprocal Recommendation}

Firstly proposed by Luiz Pizzato et al. [4], reciprocal recommender is a special kind of recommender systems. The preferences of all the users are taken into account and need to be satisfied at the same time. A similarity calculation method for calculating the reciprocal value and achieving the reciprocal recommendation based on the explicit preferences obtained from users' resumes and the implicit preferences acquired from the user's interaction history.

\section{PROPOSED SYSTEM}

Open Source Resume is a web-based application to help software development teams to recruit developers as per their skills.

This tool provides insights into developers' practical knowledge, which can be an useful criteria for judging whether they are suitable for joining the development team based on their different qualifications.

This system is a time-saver in all aspects. Also reduces the complexity. 


\section{SYSTEM INTRODUCTION}

We are developing system which is helpful for job seekers. Main advantage of this application is search is efficient. We have proposed new features which can classify category of user. User can select whether he/she is fresher or experienced. User can upload his/her resume which is shortlisted by admin according to company`s requirements. Admin will contact with H.R. executive. H. R. Will schedule interview for candidate and notify to admin. E-recruiting process is improved.

\section{SYSTEM MODULE}

\section{Candidate profile}

We have created login profile for candidate. Candidate will register to job portal by filling registration form. System will provide login ID and password to candidate. Candidate can sign in to job portal by entering given credentials. Candidate has to fill all details while registering to job portal.

\section{Admin login}

This module is designed for admin. Admin will play important role in application. Admin will also have login credential to sign into account. Admin will store all information related to candidate in database. Admin will be intermediate between candidate and H.R. company. Admin will provide all details of candidate to H.R. Similarly, Admin will provide information of company to candidate.

When candidate will apply to any job profile, admin will send candidate's resume to H.R. Candidate will upload resume which is forwarded by admin to company. When candidate enter their keyskills, admin will search suitable company profile for candidate, and convey message to candidate. If candidate attend interview then admin will update his/her status.

\section{H.R. login}

H.R module will be registered and possess credential as H.R. login. H.R. will possess information of candidate. H.R. will schedule interview for candidate. Details of further process will be maintained by H.R. There is another module i.e. company module. Candidate will search for various companies according to their requirements. All Company name and details will be stored in database.

\section{Application}

Candidate will apply through this module. Application of candidate will be stored. Number of companies and name, for which candidate is applying will be stored.

5. Interview portal

All scheduled and attained interview's details will be shown in this module.

\section{SYSTEM FEATURES}

- Efficient searching

- Reliable Review

- Proper resume building

\section{Block diagram}

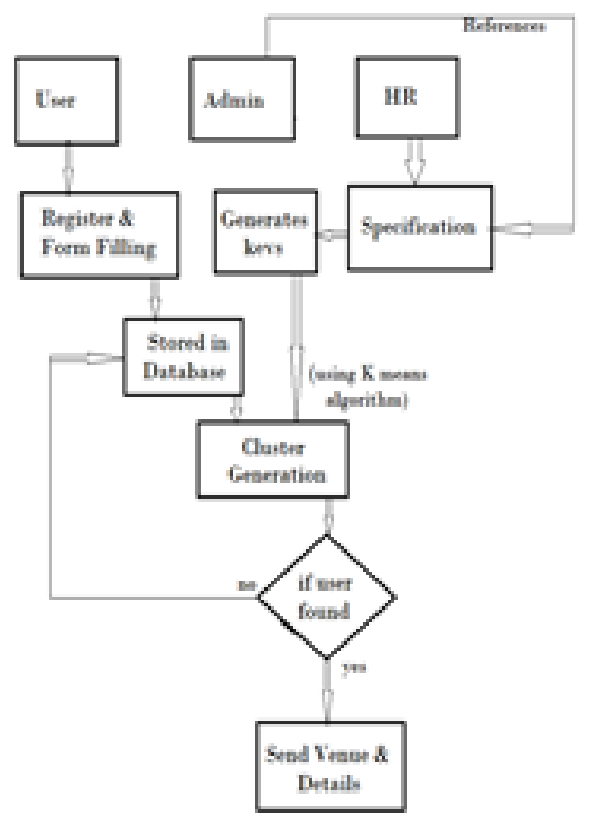

Algorithm

K-means algorithm:-

- $\mathrm{x}_{1}, \ldots, \mathrm{x}_{\mathrm{N}}$ are data points or vectors of observations

- Each observation (vector $\mathrm{x}_{\mathrm{i}}$ ) will be assigned to one and only one cluster

- C(i) denotes cluster number for the $i^{\text {th }}$ observation

- Dissimilarity measure: Euclidean distance metric

- K-means minimizes within-cluster point scatter:

$$
W(C)=\frac{1}{2} \sum_{k=1}^{K} \sum_{C(i)=k} \sum_{C(j)=k}\left\|x_{i}-x_{j}\right\|^{2}=\sum_{k=1}^{K} N_{k} \sum_{C(i)=k}\left\|x_{i}-m_{k}\right\|^{2}
$$

where

$\mathrm{m}_{\mathrm{k}}$ is the mean vector of the $\mathrm{k}^{\text {th }}$ cluster

$\mathrm{N}_{\mathrm{k}}$ is the number of observations in $\mathrm{k}^{\text {th }}$ cluster

For a given cluster assignment $\mathrm{C}$ of the data points, compute the cluster means $m_{k}$ :

$$
m_{k}=\frac{\sum_{i: C(i)=k} x_{i}}{N_{k}}, k=1, \ldots, K .
$$

For a current set of cluster means, assign each observation as:

$$
C(i)=\arg \min \left\|x_{i}-m_{k}\right\|^{2}, i=1, \ldots, N
$$

Iterate above two steps until convergence 


\section{CONCLUSION}

We presented state of the art of job recommendation as well as, a comparative study for its approaches that proposed by literatures. Additionally, we reviewed typical recommender system techniques and the recruiting process related issues. We conclude that the field of job recommendations is still unripe and require further improvements.

\section{FUTURE SCOPE}

As part of our ongoing research, we aim to build a new job recommendation approach and test with real data for employee and staffing data from large companies.

\section{REFERENCES}

1. S. T. Al-Otaibi and M. Ykhlef, "A survey of job recommender systems," International Journal of the Physical Sciences, vol. 7(29), pp. 5127-5142, July, 2012.

2. S. T. Zheng, W. X. Hong, N. Zhang and F. Yang, "Job recommender systems: a survey," In Proceedings of the $7^{\text {th }}$ International Conference on Computer Science \& Education (ICCSE 2012), pp. 920-924, Melbourne, Australia, July, 2012.

3. K. Yu, G. Guan and M. Zhou, "Resume information extraction with cascaded hybrid model," In Proceedings of the 43rd Annual Meeting of the ACL, pp. 499-506, Ann Arbor, Michigan, June, 2005.

4 I. Paparrizos, B. B. Cambazoglu and A. Gionis, "Machine learned job recommendation," In Proceedings of the fifth ACM Conference on Recommender Systems, pp. 325-328, Chicago, USA, October, 2011.

5. M. Adedoyin-Olowe, M. M. Gaber, and F. T. Stahl. A survey of datamining techniques for social media analysis. CoRR, abs/1312.4617,2013. II

6. H. W. Ye, "A Personalized Collaborative FilteringRecommendation Using Association Rules Mining andSelf-Organizing Map,” Journal of Software, vol. 6(4),pp.732-739, 2011.

7. L. Hu, W. B. Wang, F. Wang, X. L. Zhang and K. Zhao, "The Design and Implementation of CompositeCollaborative Filtering Algorithm for PersonalizedRecommendation," Journal of Software, vol. 7(9), pp.204099999999999-2045, 2012.

8. L. Pizzato, T. Rej, T. Chung, K. Yacef, I. Koprinska and J.Kay, "Reciprocal recommenders," In Proceedings of $8^{\text {th }}$ Workshop on Intelligent Techniques for Web Personalization and Recommender Systems, held inconjunction with the 18th International Conference on UserModeling, Adaptation and Personalization (UMAP 2010),Hawaii, USA, June, 2010.

9. R. Burke, "Hybrid recommender systems: survey andexperiments," User Modeling and User-Adapted

10. S. T. Al-Otaibi and M. Ykhlef, "A survey of jobrecommender systems," International Journal of thePhysical Sciences, vol. 7(29), pp. 5127-5142, July, 2012. 